University of Wollongong

Research Online

Faculty of Engineering - Papers (Archive)

Faculty of Engineering and Information

Sciences

1992

\title{
Effect of a robot's geometrical parameters on its optimal dynamic performance
}

\author{
M. Yousef Ibrahim \\ Monash University College \\ Christopher D. Cook \\ University of Wollongong, chris_cook@uow.edu.au
}

A K. Tieu

University of Wollongong, ktieu@uow.edu.au

Follow this and additional works at: https://ro.uow.edu.au/engpapers

Part of the Engineering Commons

https://ro.uow.edu.au/engpapers/4401

\section{Recommended Citation}

Ibrahim, M. Yousef; Cook, Christopher D.; and Tieu, A K.: Effect of a robot's geometrical parameters on its optimal dynamic performance 1992, 820-825.

https://ro.uow.edu.au/engpapers/4401

Research Online is the open access institutional repository for the University of Wollongong. For further information contact the UOW Library: research-pubs@uow.edu.au 


\section{EFFECT OF A ROBOT'S GEOMETRICAL PARAMETERS ON ITS OPTIMAL DYNAMIC PERFORMANCE}

\author{
M. Yousef Ibrahim \\ School of Engineering, \\ Monash University College - Gippsland, \\ Churchill, Vic. 3842, Australia.
}

\author{
C. D. Cook \\ Department of Electrical and Computer Engineering, \\ University of Wollongong, \\ Wollongong, N.S.W. 2500, Australia.
}

A. K. Tieu

Department of Mechanical Engineering, University of Wollongong, Wollongong, N.S.W. 2500, Australia.

\begin{abstract}
This paper presents a study that was conducted at Monash University College - Gippsland on the effect of a robot's geometrical parameters on its dynamic performance. This research was undertaken in pursuit of an optimal dynamic performance of industrial robots. In this study an indicator was established to quantitatively measure the dynamic performance of a robot arm with respect to changes in the geometrical parameters.

Since the dynamic behaviour of a robot's arm is largely dependent on its inertia terms; the performance indicator was based on the Logarithmic function of the sensitivity of the inertia matrix's eigenvalues to changes in a robot's geometrical parameters. Also, since the inertia matrix is a function of the joint displacement; the performance was examined for a particular parameter over a range of joint angles.

The sensitivity of the inertia matrix's eigenvalues to changes in the non-zero parameters of an articulated robot (PUMA 560) ${ }^{1}$ was examined in the course of this research. This paper presents cases related to the effect of changing the twist angles on the dynamic performance. The results of this study, which are illustrated by 3-dimensional surface plots, are discussed and analysed in this paper. All the simulation software was written in extended ANSI FORTRAN 77 and run on an HP 9000/550 computer using the UNIX operating system.
\end{abstract}

\section{Introduction}

Robot manipulators have the inherent characteristics of being highly non-linear and strongly coupled. Due to this complexity, the design of a general robot arm is an expensive and time-consuming task. Consequently, the search for the optimal dynamic performance of a robot's arm emerges as a challenging task.

This paper presents a summary of research that was recently conducted at Monash University College - Gippsland. This research was undertaken in pursuit of an optimal dynamic performance of industrial robots. In searching for optimality, the effect of different geometrical parameters was examined and analysed.

There has been considerable research to analyse the effect of a robot's geometrical parameters on its workspace performance $[1,2,3]$. However, there is no comparable work on the effect of its geometrical parameters on the dynamic performance.

In order to search for the optimal performance; a closedform computer simulation model of a general robot's dynamics was developed. The model was based on the general Lagrangian formulation. This formulation can be expressed as follows :

1 PUMA is a trade mark of UNIMATION.

$$
F_{i}=\sum_{j=1}^{N} D_{i j} \ddot{q}_{j}+\sum_{j=1}^{N} \sum_{k=1}^{N} D_{i j k} \dot{q}_{j} \dot{q}_{k}+D_{i}
$$

Where $N$ denotes the number of joints; $q, \dot{q}$ and $\ddot{q}$ denote a link's variable and its first and second time derivatives respectively, and $D_{i j}, D_{i j k}$ and $D_{i}$ are scalars associated with the inertial force term, centripetal/coriolis force term and gravitational term respectively. Details about Lagrangian formulation proof and development can be found in references [4,5].

Under normal operating conditions; a robot's dynamic performance is dominated by its inertia terms [6]. Also, the centripetal/Coriolis forces become significant only at high velocity [5]. Therefore, the sensitivity of the inertia matrix's eigenvalues to changes in a robot's geometrical parameters is taken as an indicator of performance.

Therefore, the closed-form dynamic model was further extended to include the necessary calculations for the inertia matrix's eigenvalues and its sensitivity. The study considered various geometrical parameters. The considered parameters were related to an articulated industrial robot's arm (PUMA 560).

The geometrical configuration of the PUMA 560 is schematically shown in Figure 1 and the parameters of its first three links are listed in Table 1.

Since the inertia matrix is a function of the joint variables $q_{i}(i=1, \ldots, N)$; the performance was examined for a particular parameter over a range of joint angles.

\section{Performance Measure}

In order to investigate the effect of geometrical parameters on the dynamic performance, it was necessary to establish an indicator for that performance.

Since the robot is assumed to be a three link manipulator, the dimension of the inertia matrix is $3 \times 3$. Also, $D_{i j}$ can be expressed as :

$$
D_{i j}=\sum_{p=\max (i, j)}^{N} \operatorname{Tr}\left(\frac{\partial T_{p}}{\partial q_{j}} J_{p}\left(\frac{\partial T_{p}}{\partial q_{i}}\right)^{T}\right)
$$

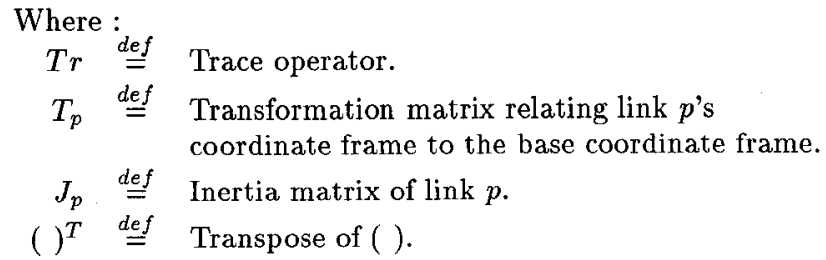




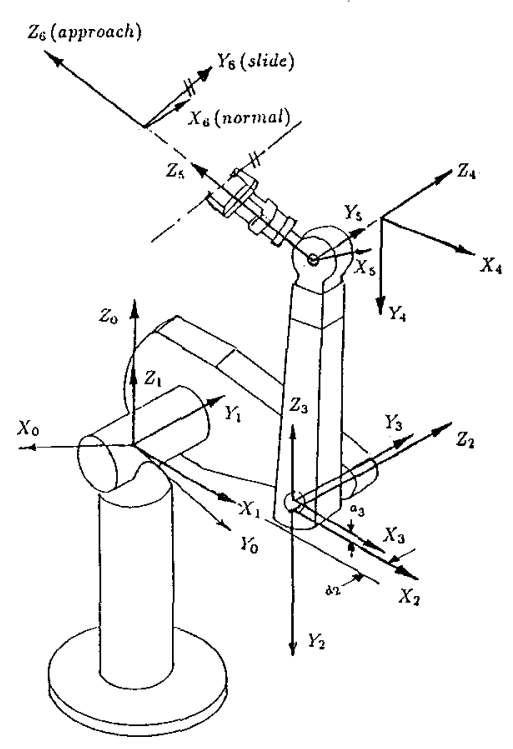

Figure 1: Schematic diagram of "PUMA 560" robot manipulator.

The inertia matrix of the three links can be expressed explicitly as follows :

$$
D(q)=\left(\begin{array}{ccc}
D_{11} & D_{12} & D_{13} \\
D_{21} & D_{22} & D_{23} \\
D_{31} & D_{32} & D_{33}
\end{array}\right)
$$

Where :

$$
\begin{aligned}
& D_{11}=\operatorname{Tr}\left(U_{11} J_{1} U_{11}^{T}\right)+\operatorname{Tr}\left(U_{21} J_{2} U_{21}^{T}\right)+ \\
& \operatorname{Tr}\left(U_{31} J_{3} U_{31}^{T}\right) \\
& D_{12}=\operatorname{Tr}\left(U_{22} J_{2} U_{21}^{T}\right)+\operatorname{Tr}\left(U_{32} J_{3} U_{31}^{T}\right) \\
& D_{13}=\operatorname{Tr}\left(U_{33} J_{3} U_{31}^{T}\right) \\
& D_{21}=\operatorname{Tr}\left(U_{21} J_{2} U_{22}^{T}\right)+\operatorname{Tr}\left(U_{31} J_{3} U_{32}^{T}\right) \\
& D_{22}=\operatorname{Tr}\left(U_{22} J_{2} U_{22}^{T}\right)+\operatorname{Tr}\left(U_{32} J_{3} U_{32}^{T}\right) \\
& D_{23}=\operatorname{Tr}\left(U_{33} J_{3} U_{32}^{T}\right) \\
& D_{31}=\operatorname{Tr}\left(U_{31} J_{3} U_{33}^{T}\right) \\
& D_{32}=\operatorname{Tr}\left(U_{32} J_{3} U_{33}^{T}\right) \\
& D_{33}=\operatorname{Tr}\left(U_{33} J_{3} U_{33}^{T}\right) \\
& U_{i j}=\frac{\partial T_{i}}{\partial q_{j}}
\end{aligned}
$$

Where :

The inertia matrix $D$ is a real symmetrical matrix due to the following facts $[7,8]$ :

$$
\begin{aligned}
& \text { 1. } \operatorname{Tr}(A)=\operatorname{Tr}(A)^{T} \quad \text { (for a square matrix } A \text { ) } \\
& \text { 2. } \operatorname{Tr}(A B C)=\operatorname{Tr}(B C A)=\operatorname{Tr}(C A B)
\end{aligned}
$$

By applying the above matrix trace characteristics in equation (2), the following relation can be obtained:

$$
D_{i j}=D_{j i}
$$

Therefore $D$ is a symmetrical matrix.

\begin{tabular}{|c||c|c|c|}
\hline \hline \multirow{2}{*}{ Parameter } & \multicolumn{3}{|c|}{ Joint } \\
\cline { 2 - 4 } & 1 & 2 & 3 \\
\hline \hline$\alpha$ & $-90^{\circ}$ & 0 & $90^{\circ}$ \\
\hline$a$ & 0 & $0.432 \mathrm{~m}$ & 0 \\
\hline$d$ & 0 & $0.149 \mathrm{~m}$ & 0 \\
\hline
\end{tabular}

Table 1: Geometrical parameters of the first three links of a "PUMA 560".

Also since every real matrix is similar to a diagonal matrix $[8]$; then $D$ is similar to a diagonal matrix. Hence, $D$ is a diagonable matrix.

Since the inertia matrix $D$ is reducible to a diagonal matrix, its characteristics values remain unchanged represented by the elements of its principal diagonal in its reduced form. Therefore, the sensitivity of the inertia matrix w.r.t. a variable can be represented by the sensitivity of its eigenvalues w.r.t. the same variable. Also, the sensitivity of the inertia matrix eigenvalues to changes in a robot's geometrical parameters is simplified by the fact that the eigenvalues of a symmetrical matrix are real $[9,10]$.

The eigenvalues of the inertia matrix in equation (3) can be expressed as :

$$
\Lambda=\left(\begin{array}{c}
\lambda_{1} \\
\lambda_{2} \\
\lambda_{3}
\end{array}\right)
$$

Also, $\xi_{i}$ was introduced to represent a robot's geometrical parameters for joint $i$, such that :

$$
\xi_{i} \in \alpha_{i}, a_{i}, d_{i}
$$

where $\alpha_{i}, a_{i}$ and $d_{i}$ denote link $i$ 's twist angle, length and offset distance respectively [5] .

In order to obtain the average response of the three eigenvalues; their Euclidean norm was calculated. This norm can be expressed as :

$$
\mu=\sqrt{\sum_{j=1}^{N} \lambda_{j}^{2}} \quad \text { (where } \mathrm{N}=\text { number of d.o.f.) }
$$

The Euclidean norm was used in order to obtain a positive average value irrespective of the sign of the individual eigenvalue. Also, since the search is focusing on finding the minimum sensitivity irrespective of the derivative's sign, the absolute values of the derivative were employed to calculate the performance indicator. This induced non-negative values for the eigenvalues' norm sensitivity. This non-negativeness of the derivative values allowed usage of their logarithm as a performance measure. Thus, a wider spectrum of the dynamic response to geometrical parameter changes became available.

Therefore, the sensitivity of an inertia matrix's eigenvalues to any change in a. link's geometrical parameter can be expressed as the derivative of their norm w.r.t. the parameter $\frac{\partial \mu}{\partial \xi_{i}} ;$ for $i=$ $1,2,3$.

Based on the above assumptions, a performance indicator for the sensitivity of the inertia matrix's eigenvalues to changes of a geometrical parameter $\xi$ of link $i$ was chosen to be $\Gamma_{\xi_{i}}$. Mathematically, the performance indicator can be expressed as follows: 


$$
\Gamma_{\xi_{i}} \triangleq f\left(\operatorname{col}(q), \xi_{i}\right)
$$

Therefore, $\Gamma_{\xi_{i}}$ can be expressed explicitly as follows :

$$
\Gamma_{\xi_{i}}=\log _{10}\left\|\frac{\partial \mu}{\partial \xi_{i}}\right\|_{(i=1, \ldots, N)}
$$

Where :

$$
\begin{aligned}
\text { Where : } & \left(\begin{array}{l}
q_{1} \\
q_{2} \\
q_{3}
\end{array}\right) \\
\mathrm{N}(q) & \stackrel{\text { def }}{=} \text { Number of joints. }
\end{aligned}
$$

A minimum sensitivity of the inertia matrix eigenvalues w.r.t. a geometrical parameter, would indicate that the parameter has a minimal influence on the dynamic performance. Also, from the point of view of adaptive control, the smaller the sensitivity of the dynamic model, the lesser the required degree of adaptation. Therefore, minimal sensitivity is desired from both dynamic optimality and control viewpoints. The sensitivity of the inertia matrix's eigenvalues to changes in the non-zero parameters of the PUMA 560 was examined in the course of this study.

The applied displacement trajectory to the links' motion was a third order polynomial function in time. In this function the position should satisfy the following relationship $[11,12]$ :

$$
\begin{aligned}
q_{t}= & (1-t)^{3}\left\{q_{0}+\left(3 q_{0}+\dot{q}_{0}\right) t+\left(\ddot{q}_{0}+6 \dot{q}_{0}+12 q_{0}\right) t^{2} / 2\right\} \\
& +t^{3}\left\{q_{1}+\left(3 q_{1}+\dot{q}_{1}\right)(1-t)\right. \\
& \left.+\left(\ddot{q}_{1}+6 \dot{q}_{1}+12 q_{1}\right)(1-t)^{2} / 2\right\}
\end{aligned}
$$

Where :

$$
\begin{aligned}
& q_{t} \stackrel{\text { def }}{=} \text { the position at time-instant }(t) \\
& q_{0} \stackrel{\text { def }}{=} \text { the initial position in space at }(t=0) \\
& q_{1} \stackrel{\text { def }}{=} \text { the final position reached at end of stroke at }(t=1)
\end{aligned}
$$
low.

The results of this study are summarised and discussed be-

\section{Effect of $\alpha_{1}$ on the Dynamic Performance}

The sensitivity of the inertia matrix's eigenvalues, $\Gamma_{\alpha_{1}}$, to the changes in the twist angle $\alpha_{1}$ was studied. The study was conducted over a range of adjacent configurations along different path trajectories. Two sets of results associated with two different motion trajectories are discussed below.

\subsection{Dynamic performance under the first motion trajectory}

In this investigation the motion displacement trajectory of the three links is schematically described in Figure 2 and its data tabulated in Table 2. The range of $\alpha_{1}$ over which the sensitivity analysis was conducted is :

$$
-90^{\circ} \leq \alpha_{2} \leq 90^{\circ}
$$

The dynamic sensitivity to changes in both $\alpha_{1}$ and robot arm configuration is shown in Figure 3 . It can be seen from this figure that the dynamic performance indicator, $\Gamma_{\alpha_{1}}$, was highly dependent on the twist angle $\alpha_{1}$. However, during the specified path trajectory illustrated in Figure 2, the changes in the robot's

\begin{tabular}{|l|c|}
\hline \hline Total displacement of link 1 & $180^{\circ}$ \\
Starting position of link 1 & $-90^{\circ}$ \\
\hline Total displacement of link 2 & 0.0 \\
Starting position of link 2 & 0.0 \\
\hline Total displacement of link 3 & $90^{\circ}$ \\
Starting position of link 3 & $90^{\circ}$ \\
\hline
\end{tabular}

Table 2: Data for the first motion trajectory for $\Gamma_{\alpha_{1}}$.

configuration had no significant effect on eigenvalues sensitivity.

It was also revealed that the lowest sensitivity is attained in the region where $\alpha_{1}=0$. Furthermore, through usage of the logarithmic function, it was found that the absolute minimum sensitivity occurs when $\alpha_{1}=0$ and $t=0$. It should be noticed that the robot's arm was fully stretched horizontally at $t=0 \mathrm{sec}$ onds through the robot's movement trajectory. That is because at that configuration (arm fully stretched horizontally) a small change in $\alpha_{1}$ would not have a significant effect on the arm's inertia due to the perpendicularity of both the second and third links to $Z_{0}$. However, that perpendicularity will not hold for any small change in $q_{2}$ or $q_{3}$. Hence, at this new configuration, any small change in the twist angle $\left(\alpha_{1}\right)$ creates a significant change in the arm's inertia.

That further explains the above-mentioned perpendicularity characteristics of a robot's links. However, where $\alpha_{1}$ is equal to zero the robot exhibits a planar motion only, thus sacrificing the workspace envelope. Therefore, a trade-off emerges between the choice of a geometrical parameter's value for optimal dynamic performance and the choice of a better workspace. Also, the dynamic performance indicator, $\Gamma_{\alpha_{1}}$, exhibited sensitivity to changes in the robot's configuration in some motion trajectories as shown in the following trajectory.

\subsection{Dynamic performance under the second mo- tion trajectory}

The eigenvalues sensitivity to $\alpha_{1}$ were examined during the course of a different robot's motion trajectory. The range of $\alpha_{1}$ was identical to that of the previous trajectory, i.e. :

$$
\alpha_{1}=-90^{\circ} \Rightarrow \alpha_{1}=90^{\circ}
$$

In this motion trajectory the second link was stationary in a

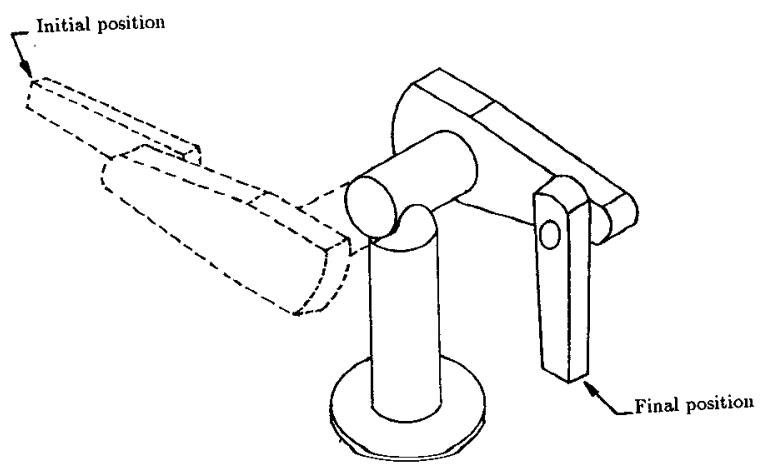

Figure 2: Schematic diagram of the robot's first path trajectory for $\Gamma_{\alpha_{1}}$. 


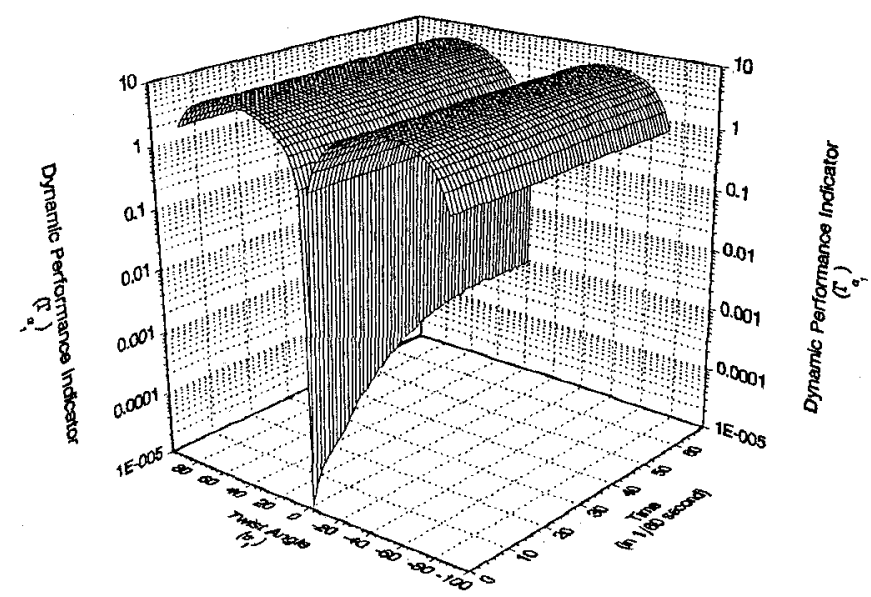

Figure 3: Dynamic performance indicator versus $\alpha_{1}$ and time (trajectory 1).

horizontal position, while the third link moved $180^{\circ}$ from $-180^{\circ}$ position (vertically downward) to $0^{\circ}$ position (vertically upward). The links' motions are schematically shown in Figure 4 and their data are summarised in Table 3. both :

Figure 5 shows the dynamic performance sensitivity $\Gamma_{\alpha_{1}}$ to

- changes in the geometrical parameter $\alpha_{1}$, and

- changes in the robot's configuration through the motion trajectory.

From the plotted results in Figure 5, the eigenvalues sensitivity was again smaller about the zero value of $\alpha_{1}$. It was also found that the sensitivity is symmetrical about $t=0.5$ seconds. That was due to the symmetry of the robot's path trajectory below and above $q_{3}=90^{\circ}$.

It is found from $\Gamma_{\alpha_{1}}$, shown in Figure 5, that the absolute minimum sensitivity occurred at $t=0.5$ seconds. It should be noticed that the robot's arm was fully stretched horizontally at this point in time through the robot's movement trajectory. That further validates the perpendicularity characteristic of a robot's links mentioned in section 3.1 .

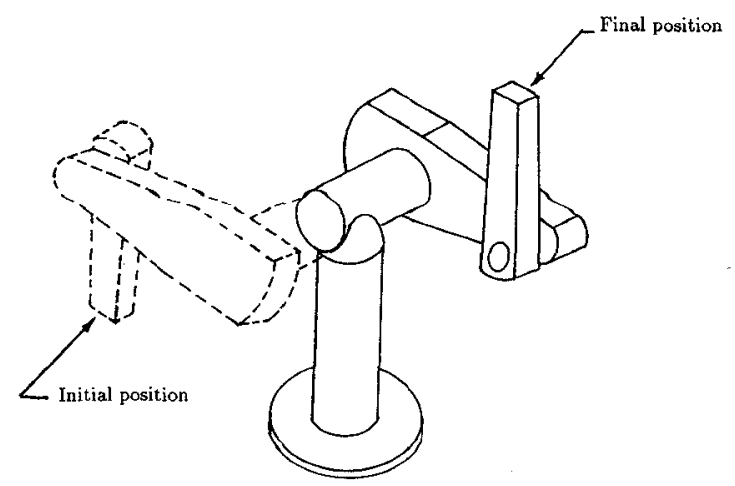

Figure 4: Schematic diagram of the robot's second path trajectory for $\Gamma_{\alpha_{1}}$.

\begin{tabular}{|l|c|}
\hline \hline Total displacement of link 1 & $180^{\circ}$ \\
Starting position of link 1 & $-90^{\circ}$ \\
\hline Total displacement of link 2 & 0.0 \\
Starting position of link 2 & 0.0 \\
\hline Total displacement of link 3 & $-180^{\circ}$ \\
Starting position of link 3 & $180^{\circ}$ \\
\hline
\end{tabular}

Table 3: Data for the second motion trajectory for $\Gamma_{\alpha_{1}}$.

\section{Effect of $\alpha_{2}$ on the Dynamic Performance}

Further simulation experiments were conducted to investigate the effect of the second link's twist angle $\alpha_{2}$ on the dynamic performance. The experiments were conducted for two different motion trajectories. In the first motion trajectory the robot's arm was stretched horizontally and rotated $180^{\circ}$ in a horizontal plane around $Z_{0}$. In the second trajectory the robot's arm was also stretched during the motion's cycle and moved $180^{\circ}$ about $Z_{1}$.

\subsection{Dynamic performance under the first motion trajectory}

The sensitivity of the inertia matrix eigenvalues $\left(\Gamma_{\alpha_{2}}\right)$, was examined for different values of the twist angle $\alpha_{2}$. The range of $\alpha_{2}$ was as follows:

$$
-90^{\circ} \leq \alpha_{2} \leq 90^{\circ}
$$

Also the robot's path trajectory of this case is schematically described in Figure 6 and its data tabulated in Table 4.

Since the arm was rotating fully stretched horizontally in a planar motion, the dynamic performance indicator was affected mainly by the changes in $\alpha_{2}$. Also, Figure 7 shows that there were sudden changes in the eigenvalues derivatives about $\alpha_{2}=0$. That was because the arm was fully stretched throughout the cycle. Therefore, any change in $\alpha_{2}$ causes a significant reaction in the dynamic sensitivity due to the perpendicularity mentioned in section 3.1. Also it appears from the plotted results that at $\alpha_{2}= \pm 45^{\circ}$ the sensitivity was consistently high. Furthermore, throughout the motion trajectory the arm was, theoretically, at

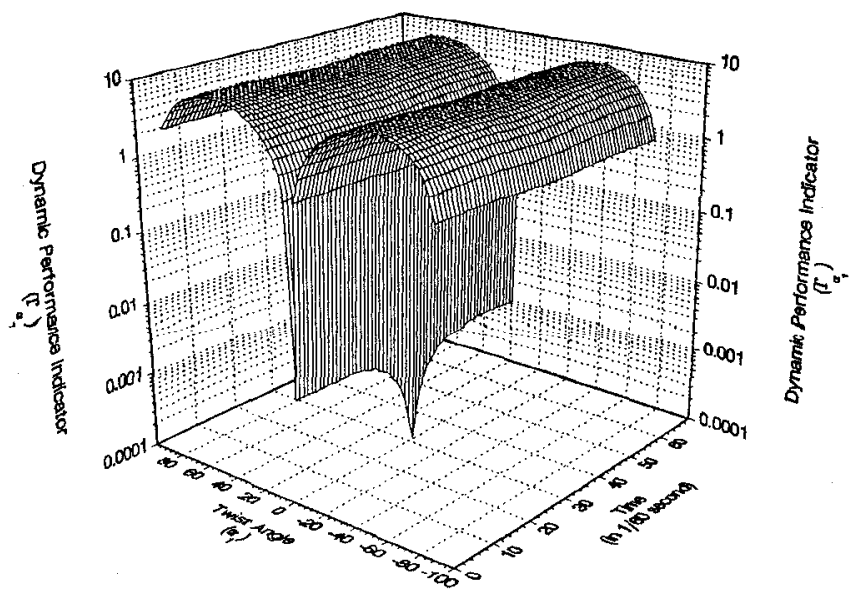

Figure 5: Dynamic performance indicator versus $\alpha_{1}$ and time (trajectory 2). 


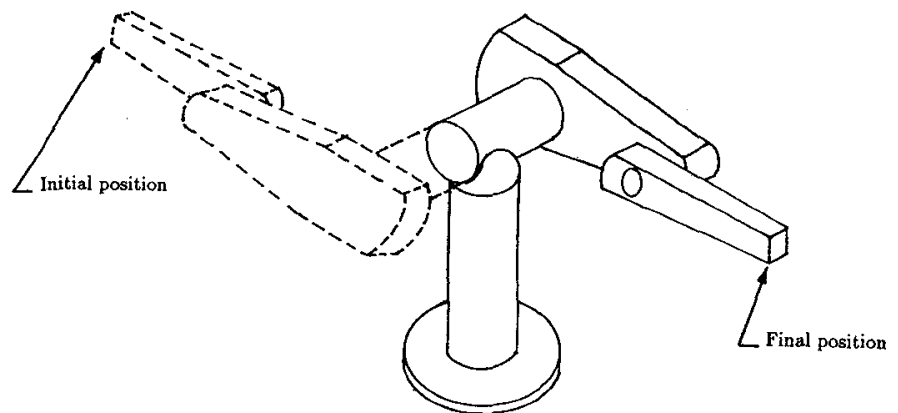

Figure 6: Schematic diagram of the robot's first path trajectory for $\Gamma_{\alpha_{2}}$.

singular positions. It is clear that the optimal value of $\alpha_{2}$ is zero.

Also, the sensitivity's trend is generally uniform along the trajectory in which both $q_{2}$ and $q_{3}$ are constant, as demonstrated by the sensitivity shown in Figure 7 .

\subsection{Dynamic performance under the second mo- tion trajectory}

In the second motion trajectory the first and second links were moving concurrently, each for $180^{\circ}$. During this trajectory the arm was continually stretched and moved from a vertically downward position to a vertically upward position. The motion's trajectory is schematically described in Figure 8 and its data are tabulated in Table 5 .

In this case, the minimum sensitivity was attained under the following conditions :

- When $\alpha_{2}=0$.

- When $t=0.5$ seconds.

A high rate of change in the robot's sensitivity $\Gamma_{\alpha_{2}}$ occurred immediately before and after the above-mentioned values for $\alpha_{2}$ and time $t$, as shown in Figure 9. Also, in this motion's path trajectory the arm was fully stretched horizontally at $t=0.5$ seconds.

Also, it was revealed from Figure 9 that the minimum sensitivity occurs at $\alpha_{2}=0$.

The results shown in this experiment make it possible for a robot designer to choose the optimal workspace region together with suitable twist angles in order to obtain a robot's best possible dynamic performance.

\begin{tabular}{|l|c|}
\hline \hline Total displacement of link 1 & $180^{\circ}$ \\
Starting position of link 1 & $-90^{\circ}$ \\
\hline Total displacement of link 2 & 0.0 \\
Starting position of link 2 & 0.0 \\
\hline Total displacement of link 3 & $0.0^{\circ}$ \\
Starting position of link 3 & $90^{\circ}$ \\
\hline
\end{tabular}

Table 4: Data for the first motion trajectory for $\Gamma_{\alpha_{2}}$.

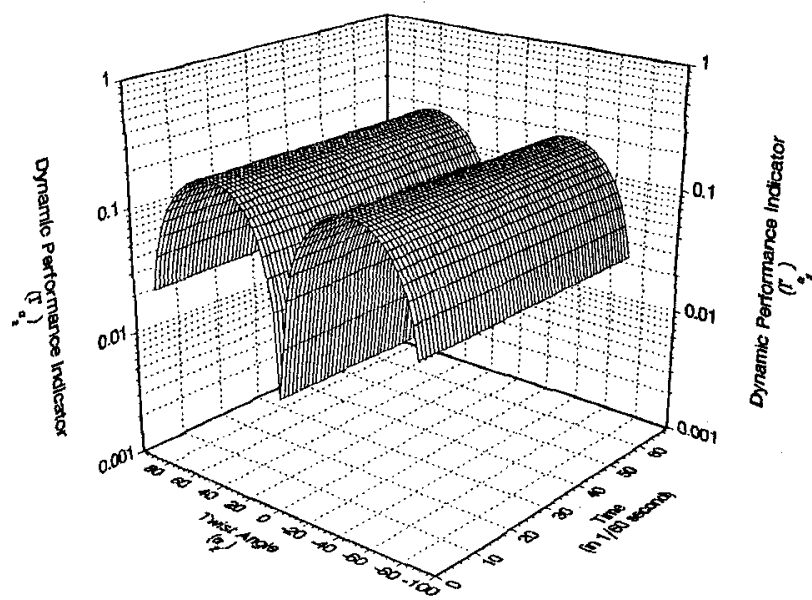

Figure 7: Dynamic performance indicator versus $\alpha_{2}$ and time (trajectory 1).

\section{Summary}

In this paper a study was conducted to investigate the effect of a robot's geometrical parameters on its dynamic performance. Therefore, a performance measure indicator was introducedeto give a quantifiable measure of the dynamic performance's. sponse to changes in the links' geometrical parameters. The dynamic performance indicator was based on the logarithm of the Euclidean norm of the inertia matrix eigenvalue derivatives with respect to the geometrical parameter under consideration. That was achieved taking advantage of the symmetrical property of the inertia matrix.

The search was for a region in which there was minimum sensitivity to parameter changes over a specified path. As shown

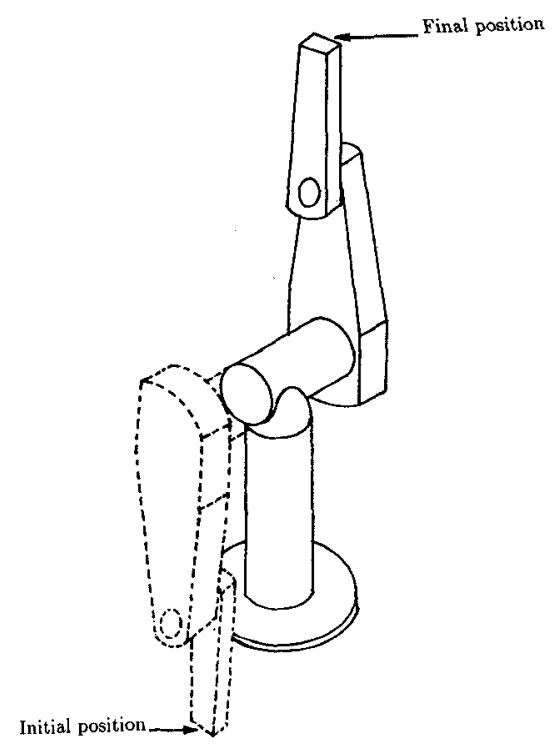

Figure 8: Schematic diagram of the robot's second path trajectory for $\Gamma_{\alpha_{2}}$. 


\begin{tabular}{|l|c|}
\hline \hline Total displacement of link 1 & $180^{\circ}$ \\
Starting position of link 1 & $-90^{\circ}$ \\
\hline Total displacement of link 2 & $-180^{\circ}$ \\
Starting position of link 2 & $90^{\circ}$ \\
\hline Total displacement of link 3 & $0.0^{\circ}$ \\
Starting position of link 3 & $90^{\circ}$ \\
\hline
\end{tabular}

Table 5: Data for the second motion trajectory for $\Gamma_{\alpha_{2}}$.

from the obtained results, large variations in the performance indicator do not, necessarily, mean a poor design (from a workspace perspective). However, an important fact has been unveiled through this research, that is, a design of a robot for optimal dynamic performance can be achieved by taking into account two constraints :

1. geometrical parameter values such that the robot would have its necessary workspace envelope to execute its required task.

2. path constraints such that the robot would move through a low sensitivity corridor in its work envelope.

As demonstrated graphically through the logarithmic plots of the cases studied, the performance sensitivity is greater to changes in the twist angles than to changes in the robot's configuration. Also, It was consistently found that smaller twist angles lead to a better performance criteria. Therefore it was recommended to minimise the twist angles where practicable. This has prompted the notion for a new breed of robots with adjustable twist angles.

This new general design mechanism was established during the course of this research to give a robot designer a quantitative feedback, with graphical illustration, regarding the influence of changing a robot's geometrical parameters on its optimal dynamic performance.

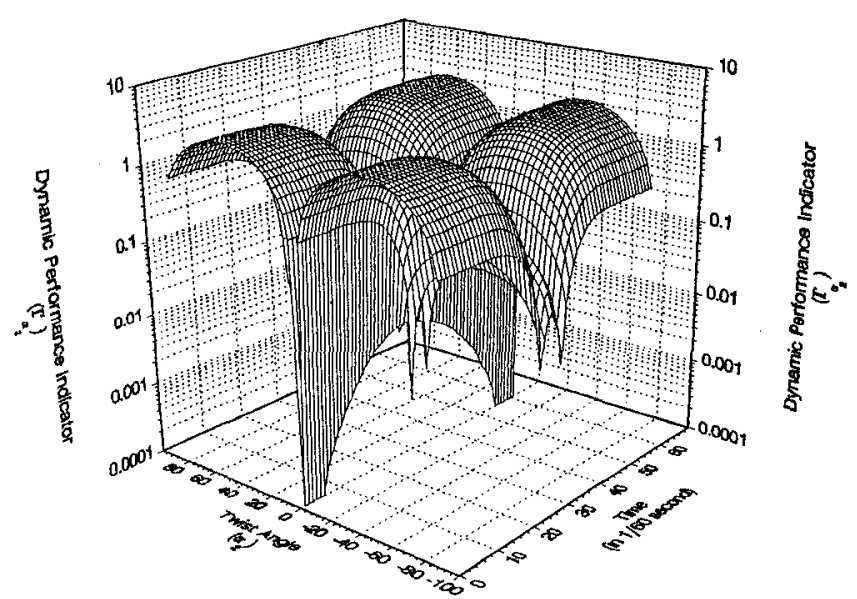

Figure 9: Dynamic performance indicator versus $\alpha_{2}$ and time (trajectory 2).

\section{References}

[1] Y. C. Tsai and A. H. Soni. The effect of link parameter on the working space of general $3 \mathrm{R}$ robot arms. Mechanism and Machine Theory, 19(1):9-16, 1984.

[2] K. C. Gupta and B. Roth. Design considerations for manipulator workspace. ASME Journal of Mechanical Design, 104:704-711, October 1982.

[3] Tsuneo Yoshikawa. Dynamic manipulability of robot manipulators. Journal of Robotics Systems, 2(1):113-124, 1985.

[4] A.K. Bejczy. Robot arm dynamics and control. Technical Report 33-669, Jet Propusion Lab, Pasedena, CA, February 1974.

[5] Richard P. Paul. Robot Manipulators: Mathematics, Programming and Control. MIT Press, Cambridge, MA, 1981.

[6] M. Y. Ibrahim, C. D. Cook, and A. K. Tieu. Dynamic behaviour of a SCARA robot with links subjected to different velocity trajectories. Robotica, 6:115-121, 1988.

[7] F.M. Stein. Introduction to Matrices and Determinants. Wadsworth, Belmont, California, 1967.

[8] H. Schneider and G.P. Barker. Matrices and Linear Algebra. Holt, Rinehart and Winston, New York, 1973.

[9] Erwin Kreyszig. Advanced Engineering Mathematics. John Wiley, New York, 1972.

[10] P.V. O'Neil. Advanced Engineering Mathematics. Wadsworth, Belmont, California, 1983.

[11] M. S. Mujtoba. Discussion of Trajectory Calculation Methods. Technical Report AIM 285.4, Stanford University, Artificial Intelligence Laboratory, 1977.

[12] Michael Brady, John M. Hollerbach, Timothy L. Johnson, Thomas Lozano-Perez, and Matthew T. Mason. Robot Motion: Planning and Control. MIT Press, Cambridge, MA, 1982 . 